\title{
Numerical analysis of flood with a double grid model
}

\author{
Go Morikawa $^{1,{ }^{*}}$ and Ichiro Kimura ${ }^{2}$ \\ ${ }^{1}$ Graduate School of Engneering, Hokkaido University, Hydraulic Research Laboratory, Japan \\ ${ }^{2}$ Faculty of Engineering, University of Toyama, Japan
}

\begin{abstract}
The objective of this study is to verify the validity of a hyper grid type (double grid model) computational model, for calculating flood flows with inundations. Nowadays, detailed information of the bathymetry is available due to improved measurement techniques, such as LP data. However, the number of grid cells used in the computation is limited because of the limitation of computational infrastructures. A double grid approach based on the model proposed by Volp et. al.(2013) is employed to overcome this problem. This model directly uses the high resolution topographic data though the discretization of the governing equations are made on coarser grid. The computational results showed that the present numerical model can compute the large scale flood phenomena efficiently and accurately.
\end{abstract}

\section{Background}

Heavy rains like Guerrilla rainstorms often happen recently in Japan because of climate change. Generally, Japanese rivers' slopes are steeper and the lengths are shorter than rivers in a continent. Therefore, heavy rains sometimes cause huge floods in Japan. That is the reason why fast and accurate simulation method for flood with inundation is necessary.

Nowadays, detailed information of the bathymetry is available because of improved measurement techniques ${ }^{1)}$. Bathymetric data with a very high spatial resolution of the order of meters become available and are usually easily obtained through web-site. Generally, the results of computations that use such detailed bathymetric data are more accurate than the results with coarse data. However, CPU time of the computation increases if such highresolution data are used. ${ }^{2)}$ To overcome this dilemma, Volp et al. (2013) proposed a hyper grid (double grid) approach, in which bathymetric data are stored in very fine grids though the computations are made in much coarse grids ${ }^{2}$. This kind of double grid models were also proposed by some other researcher, but the model proposed by Volp et al. employs the Cartesian type grid, thus the governing equations become very simple and the computational time is expected to be smaller than other models. Therefore, we try to apply it to some different types of flood phenomena with inundation in this paper.

Two cases of flood phenomena are tested in this study. One is for a simple topography with two-dimensional periodic wavy bed forms. The other case is on a real river case. We employed the flood at upstream reach in the Toyohira River, Sapporo, Japan.

* Corresponding author:go-go@eis.hokudai.ac.jp 
Through examinations of the computational results in two different cases, it is shown that the present model is an efficient and accurate computational tool to simulate large scale flood phenomena with inundation.

\section{Computational model}

We employed a double grid type simulation model for flood developed by Volp et al.(2013) ${ }^{2)}$. The model uses two types of grids: one is a coarse computational grid on which the governing equations are discretized and the other is a fine grid called subgrid, which stores the bathymetric data such as bed elevation and roughness coefficient. This model assumes that water level within a coarse grid cell is uniform. Governing equations are solved on a coarse grid, but the information on the finer subgrid is reflected on the variables on the coarse grid. In this process, the advection terms and bed friction terms in the governing equations are integrated on a coarse grid considering the fine grid bathymetric information, and thus the discretized forms of the finite volume equations are obtained. The detailed computational process is explained by Volp et al. $(2013)^{2)}$ and Ali et al. $(2016)^{3)}$.

As for the inlet boundary conditions, the discharge was specified considering the hydrograph and the water depth at the inlet boundary was determined by the Manning equation. At the outlet boundary, the gradient zero conditions were specified for all hydraulic variables ${ }^{3)}$. Those conditions were employed by considering the matching with the physical phenomena and the stability of the computations.

\section{Computational conditions}

\subsection{Tested three models}

We calculated the flow by three different numerical models. One is the double grid type model. Another model is a single model, which grid size is same with the fine grid in the double grid type model. The other one is a single grid model again, but the size of the grid is set a little bit smaller than the coarse grid in the double grid type model.

\subsection{Bathymetric topography}

We tested two types of bathymetric topography (Cases 1 and 2). Case 1 employs a simple periodically waved bed form shown in Figure 1. Case 2 employs a topography data from a real river basin of the upstream reach of the Toyohira River, Sapporo, Japan. Figure 2 shows the aerial photograph around the area of Case 2 and Figure 3 shows the bird's-eye view of the bathymetric topography of Case 2 .

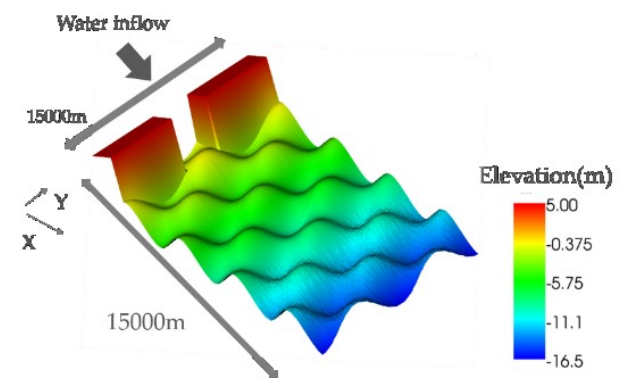

Fig. 1. The bird's-eye view of the bed topography in Case 1 . 


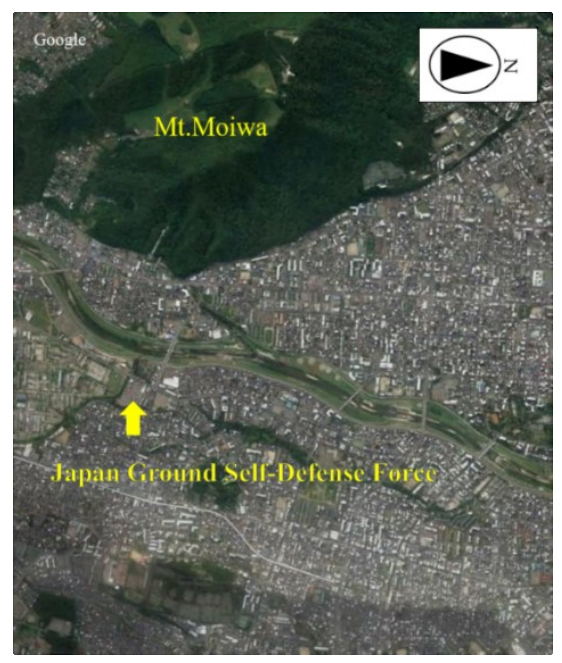

Fig. 2. The aerial photograph around the computational area in Case 2.

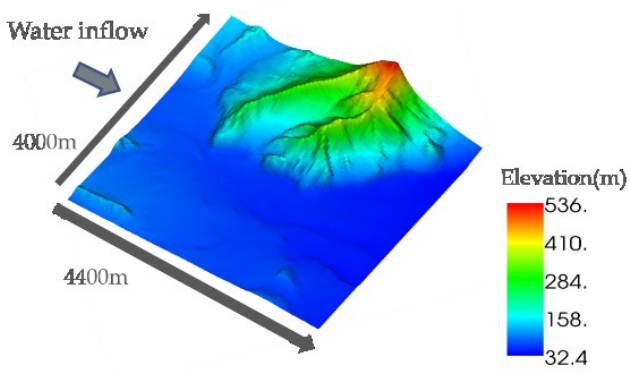

Fig. 3. The bird's-eye view of the bed topography in Case 2 .

\subsection{Calculation condition}

Table 1 shows the calculation conditions for both Cases 1 and 2. In Case 1, there are $100 \times 100$ subgrids and $10 \times 10$ coarse grids for the double grid model. The shape of grid cells for both subgrid and computational grid is square. The edge length of the subgrid cell and computational cell are $150[\mathrm{~m}]$ and $1500[\mathrm{~m}]$, respectively. Therefore, one coarse grid cell is divided into $10 \times 10$ subgrid cells. In the single fine grid and the single coarse grid model, we divide the computational area into $100 \times 100$ fine grid cells and $20 \times 20$ coarse grid cells, respectively. The average gradient of the computational area is $1 / 1000$. The roughness is assumed to be uniform with the Manning roughness coefficient of 0.05 . Flow discharge is set $4000\left[\mathrm{~m}^{3} / \mathrm{s}\right]$ (constant discharge, the shape of the hydrograph is flat).

In Case 2, we divide the computational area in the same way with Case 1 . We do not have detailed information of the values of Manning roughness coefficient in this area. Therefore, we set the Manning roughness coefficient 0.03 uniformly. The reason is that the most of expected inundation area in Case 2 is urbanized and the normal value for the urbanized area is expected to be 0.03 . This is a little bit rough approach, but it may be acceptable because the main purpose of this computation is not simulating a real flood but model comparisons. The discharge is set constant again $\left(=2000 \mathrm{~m}^{3} / \mathrm{s}\right)$ for simplicity.

In both cases, the total calculation time is set 176400 [sec] considering the enough time to reach the equilibrium state. 
Table 1. The calculation condition

\begin{tabular}{|c|c|c|c|c|}
\hline & \multicolumn{2}{|c|}{ Case 1} & \multicolumn{2}{c|}{ Case 2 } \\
\hline $\begin{array}{c}\text { Number of grid cells } \\
\text { in the double grid } \\
\text { model[edge length] }\end{array}$ & $\begin{array}{c}100 \times 100 \\
{[150 \mathrm{~m} \times 150 \mathrm{~m}]}\end{array}$ & $\begin{array}{c}10 \times 10 \\
{[1500 \mathrm{~m} \times 1500 \mathrm{~m}]}\end{array}$ & $\begin{array}{c}100 \times 100 \\
{[40 \mathrm{~m} \times 44 \mathrm{~m}]}\end{array}$ & $\begin{array}{c}10 \times 10 \\
{[400 \mathrm{~m} \times 440 \mathrm{~m}]}\end{array}$ \\
\hline Single fine grid & \multicolumn{2}{|c|}{$100 \times 100[150 \mathrm{~m} \times 150 \mathrm{~m}]$} & $100 \times 100[40 \mathrm{~m} \times 44 \mathrm{~m}]$ \\
\hline Single coarse grid & $20 \times 20 \quad[750 \mathrm{~m} \times 750 \mathrm{~m}]$ & $20 \times 20 \quad[200 \mathrm{~m} \times 220 \mathrm{~m}]$ \\
\hline Discharge & \multicolumn{2}{|c|}{$4000\left[\mathrm{~m}^{3} / \mathrm{s}\right]$} & $2000\left[\mathrm{~m}^{3} / \mathrm{s}\right]$ \\
\hline Mannig roughness & \multicolumn{2}{|c|}{0.05} & 0.03 \\
\hline Average bed slope & \multicolumn{2}{|c|}{$1 / 1000$} & \multicolumn{2}{c|}{} \\
\hline Total calculation time & \multicolumn{2}{|c|}{$176400[\mathrm{sec}]$} \\
\hline
\end{tabular}

\section{The calculation result}

The computational times(CPU time) of in the double grid model and single fine grid model with Intel(R)Core(TM)i5-6200U are listed in Table 2.

In Case 1, the CPU time for the double grid model and the single fine grid model is 99 [sec] and 287 [sec], respectively. The CPU time with the double grid model is $66 \%$ shorter than the time with the single fine grid model.

In Case 2, the CPU time for the double grid model and the single fine grid model is 300 [sec] and 2540 [sec], respectively. The necessary time for computation with the double grid model is $88 \%$ shorter than the time with the single fine grid model.

Through these results, we can expect that the computational efficiency for the double grid model becomes more outstanding.

Table 2. The comparison of computational time

\begin{tabular}{|c|c|c|c|}
\hline & Double grid model & Single fine Grid & \\
\hline Case 1 & $99[\mathrm{sec}]$ & $287[\mathrm{sec}]$ & $66 \%$ shorter \\
\hline Case 2 & $300[\mathrm{sec}]$ & $2540[\mathrm{sec}]$ & $88 \%$ shorter \\
\hline
\end{tabular}

Figure 4 shows the comparison of color contours for the simulated water depth in the results of Case1. The result with the double grid model and the result with the single fine grid model are similar although the profile of the depth by the double grid model is not smooth compared with the result by the single fine grid model. The result with the single coarse grid model considerably underestimated the water depth.

The profiles of the water depth along the line at $y=7500[\mathrm{~m}]$ in Case 1 are drawn in Figure 5. The maximum depths at every bed convex are listed in Table 3 . From Table 3, the computed depth at each convex part by the double grid model shows similar values with the result by the single fine grid model, while the result with the single coarse grid model underestimated the maximum depth at the bed convex parts.

In Figure 5, we can find that the depth at $x=1500[\mathrm{~m}], 6000[\mathrm{~m}], 9000[\mathrm{~m}]$ and 13500 $[\mathrm{m}]$ are $0[\mathrm{~m}]$ (dry cell) in the result with the double grid model. These points locate at the boundaries of the coarse grid cells. 


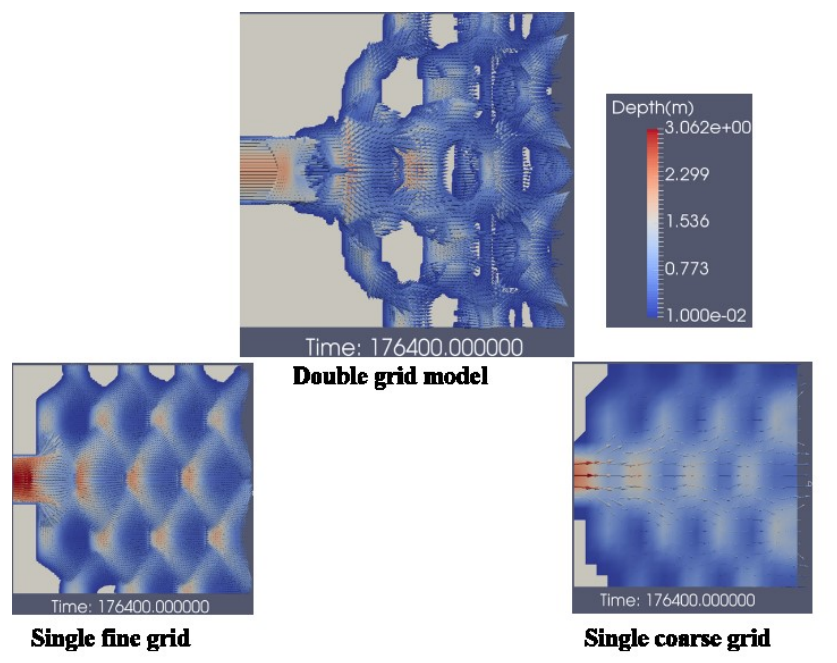

Fig. 4. The color contour maps of water depth in the results of Case 1.

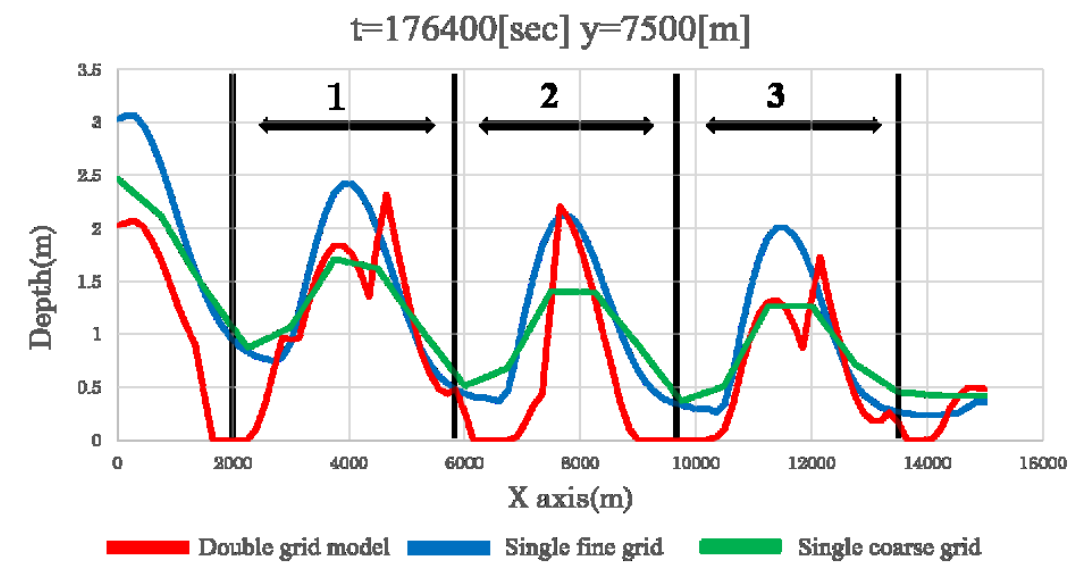

Fig. 5. The depth of Case 1 in the longitudinal section at $y=7500[\mathrm{~m}]$.

Table 3. The maximum depth at every convex part of bed topography.

\begin{tabular}{|c|c|c|c|}
\hline & 1 & 2 & 3 \\
\hline Double grid model & $2.32[\mathrm{~m}]$ & $2.20[\mathrm{~m}]$ & $1.73[\mathrm{~m}]$ \\
\hline Single fine grid & $2.42[\mathrm{~m}]$ & $2.12[\mathrm{~m}]$ & $2.01[\mathrm{~m}]$ \\
\hline Single coarse grid & $1.71[\mathrm{~m}]$ & $1.41[\mathrm{~m}]$ & $1.26[\mathrm{~m}]$ \\
\hline
\end{tabular}

Figure 6 shows the color contours of the simulated water depth in the results of Case 2 . Similar to the results in Case 1, the result by the double grid model shows similar pattern with the result by the single fine grid though the result by the single coarse grid partially underestimated the water depth.

Figure 7 shows the color contour of the water depth with double grid model at the end of the computation. In the Figure 7, the yellow line represents the section at $y=2000[\mathrm{~m}]$ and the blue line represents the section at $\mathrm{y}=1600[\mathrm{~m}]$. Figure 8 shows the depth in the 
longitudinal sections at $y=2000[\mathrm{~m}]$ and $1600[\mathrm{~m}]$. In this figure, it is easy to understand that the depth profiles with double grid model and with the single fine grid model are very similar, though the result by single coarse grid model is different from the results by other two models.

We can also find a clear error in the result with double grid model. The orange line in Figure. 7 represents lateral section at $x=4400[\mathrm{~m}]$. Figure 9 shows the depth profile along this line. The figure shows that the simulated depth around $x=4400[\mathrm{~m}]$. There are large discrepancy of the simulated depthes between the results by the double grid model and the single fine grid model at $\mathrm{y}=3000[\mathrm{~m}]$. Figure. 10 shows that the bed elevation around $\mathrm{x}=$ $4400[\mathrm{~m}]$ and it changes suddenly around $\mathrm{y}=3000[\mathrm{~m}]$, while water level keeps almost flat there. That means the flow to the small left bifuraction is overestimated in the double grid model. We can expect that the error is caused by the assumption that the water level is uniform at each coarse grid cell in the double grid model. This assumption may cause a locally large error if the bathymetric profile is complicated. This result indicates the necessity of model refinement around the portions with sudden change of bed level. We confirmed that the global mass conservation is completely satisfied in this model because we checked that inlet and outlet discharge is identical under the equiribrium state.

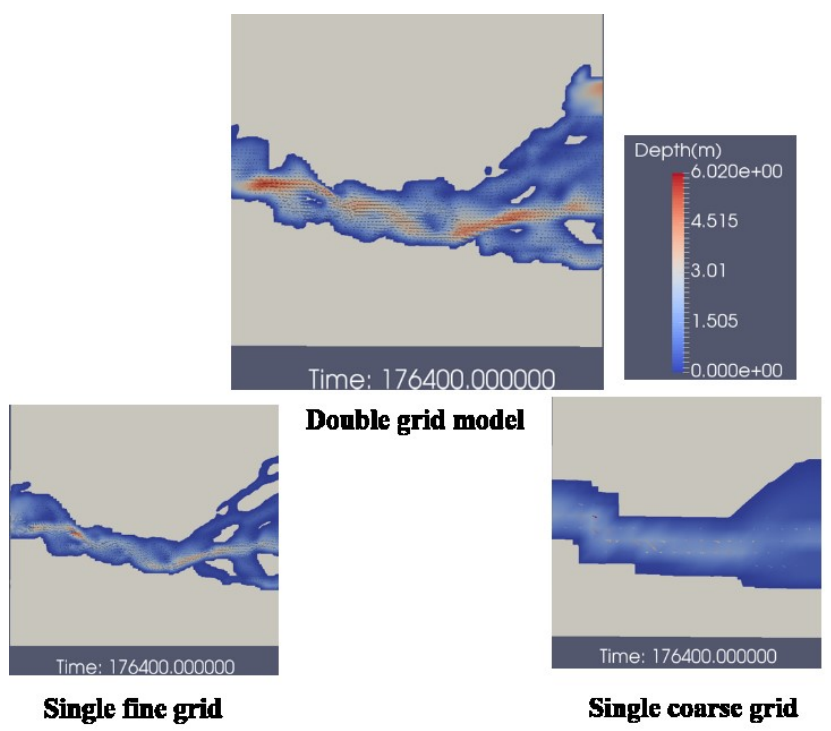

Fig. 6. The color contour map of the water depth in the results of Case 2.

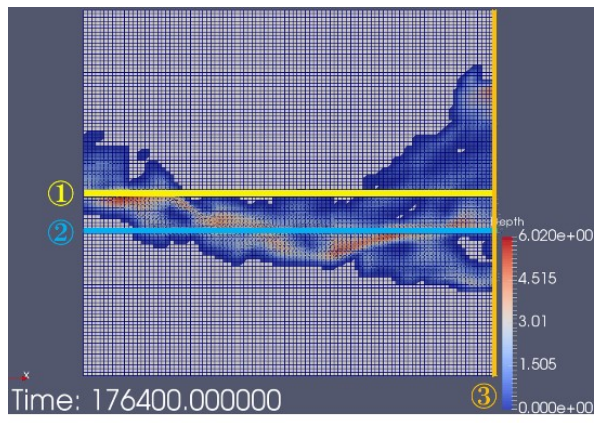

Fig. 7. The color contour map of water depth in the result with the double grid model. 

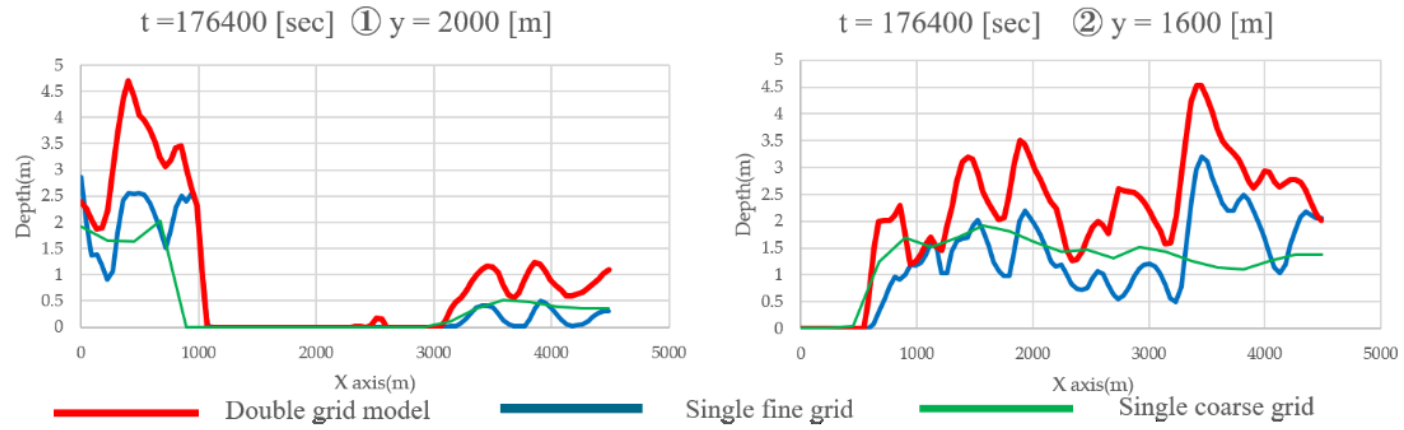

Fig. 8. The depth profiles in the streamwise longitudinal sections at $y=2000[\mathrm{~m}]$ and $1600[\mathrm{~m}]$.

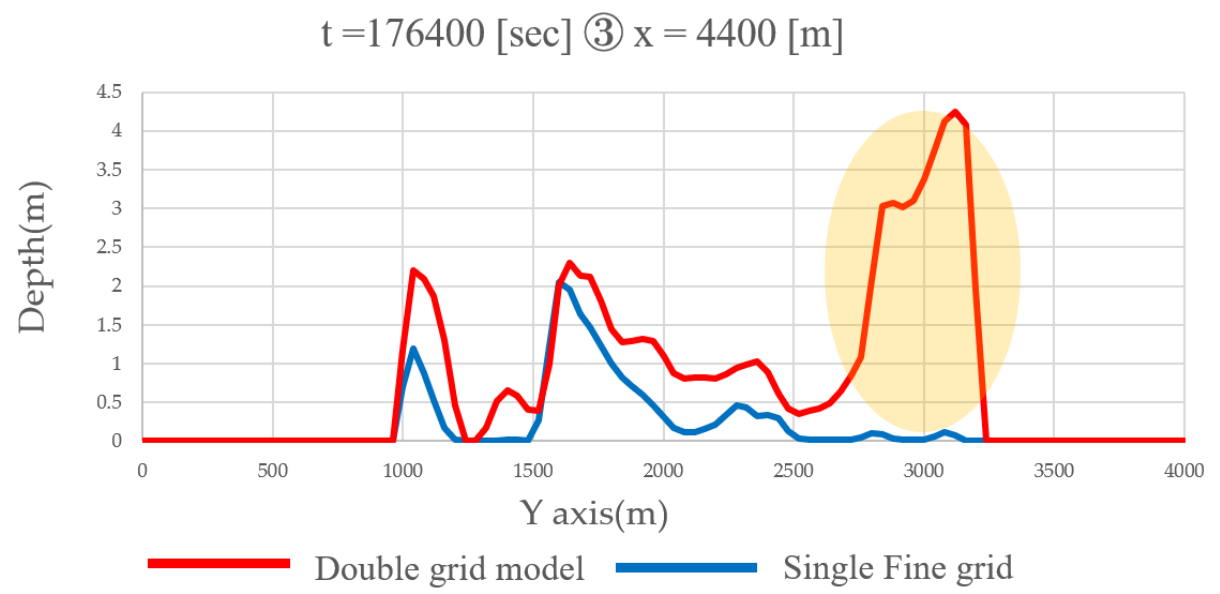

Fig. 9. The depth profiles along the cross-section at $x=4400[\mathrm{~m}]$. 


\section{$\mathrm{t}=176400[\mathrm{sec}]$ (3) $\mathrm{x}=4400[\mathrm{~m}]$ (Double grid model)}

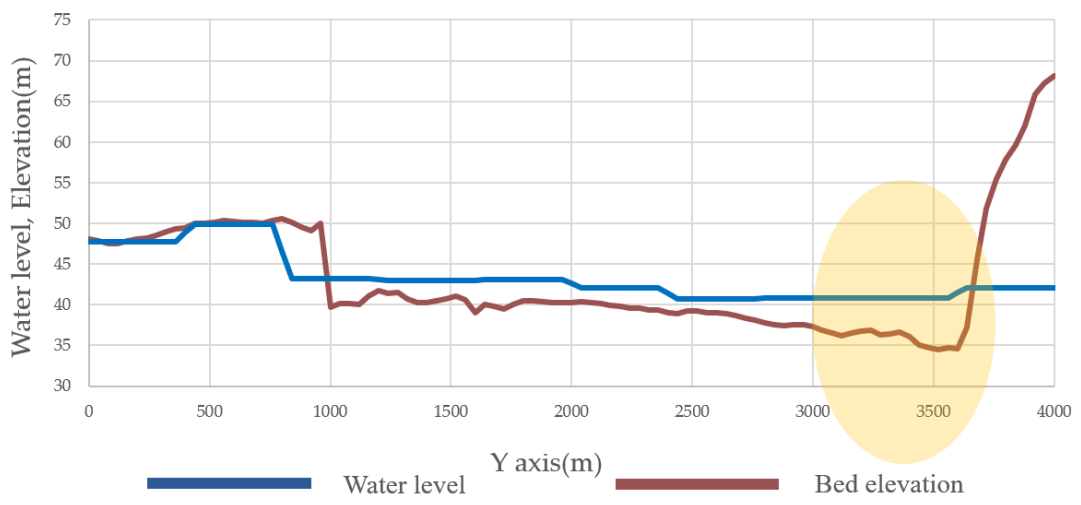

Fig. 10. The bed elevation and waterlevel along the cross-section at $x=4400[\mathrm{~m}]$.

\section{Summary}

In this paper, we verified the validity of the double grid model proposed by Volp et al. (2013), which employs double grid approaches coupling coarse computational grid and fine topography grid. We tried to apply this model to flood phenomena with inundation and examine the accuracy and computational efficiency. The main findings of the present study are summerized as follows.

1. The result by the presnet double grid approach is competitive with the result with the single fine grid model under the same resolution of the bathymetric data, though the computational time with the double grid model is much shorter than the time with the single fine grid model.

2. Accuracy of the double grid model becomes relatively worse where the bed elevation changes suddenly. The reason is that the double grid model assumes that the water level in a coarse grid cell is uniform. It implies the necessity of further model refinement.

\section{References}

1) Shin Miura, Ikuo Kawamura, Ichiro Kimura, Atsuyoshi Miura: Study on inundation flow analysis method in densely populated urban areaon alluvial fan, Proceedings of hydraulic engineering, JSCE, pp. S_979-S_984, (2011)

2) N. D. Volp, B. C. van Prooijen, G. S. Stelling:A finite volume approach for shallow water flow accounting for high-resolution bathymetry and roughness data, WATER RESOURCES RESEARCH, VOL. 49, 4126-4135, (2013)

3) Malik Ahmad Ali, Ichiro Kimura \& Yasuyuki Shimizu: Flood modelling using sub-grid based finite volume approach \& constrained interpolation profile method, River Flow 2016 - Constantinescu, Garcia \& Hanes (Eds), pp. 1891-1895, (2016) 